\title{
Incorrect citation
}

T his scenario was based around having an off-site attending veterinarian (AV) to service the veterinary care of Reddington State University (RSU), but could also be applicable to situations where the AV is a full-time staff member. We believe RSU should appeal the citation for several reasons. It is stated that RSU has a written program of veterinary care and is approved by the USDA, therefore, the citation appears to have been issued based on the choice of clinical care and a principal investigator (PI) decision to euthanize the pig.

The Animal Welfare Act Regulations ${ }^{1}$, the Guide ${ }^{2}$, and $\mathrm{ACLAM}^{3}$ all state that a facility shall provide adequate veterinary care and state further that there should

\section{A WORD FROM USDA}

The United States Department of Agriculture's Animal Care program appreciates the opportunity to respond to this scenario. First, we wish to make clear that our focus during an inspection is on assessing the compliance of a facility with the Animal Welfare Act and regulations. It is not customary for an inspector to attribute a facility's failure to seek appropriate follow up care to an attending veterinarian or to accuse a medical professional of negligence in an inspection report. Accordingly, we do not believe this fictitious scenario accurately depicts the USDA inspection process.

A facility utilizing a part-time attending veterinarian to provide regular visits fulfills the regulatory requirements for an attending veterinarian ${ }^{1}$. The regulations under 9 CFR $\$ 2.33$ - Attending veterinarian and adequate veterinary care require: appropriate methods to treat injuries, daily observations to assess animal health and well-being, guidance to personnel involved in the care of the animals, and adequate preand post-procedural care in accordance with established veterinary medical and nursing procedures ${ }^{2}$. In regards to daily observations, if not performed by the attending veterinarian, a mechanism of direct and frequent communication is required so that accurate information on problems can be conveyed to the attending veterinarian in a timely manner ${ }^{3}$. be timely and accurate methods for communication of any abnormalities in or concerns about animal health, behavior, and well-being to the veterinarian. According to the USDA Animal Welfare Inspection Guide ${ }^{4}$, the facility should be considered in compliance if: the veterinary care issue was identified by the facility prior to inspection; and the facility followed the authorized treatment plan or contacted the $\mathrm{AV}$; and the treatment plan was followed but does not appear to be effective and the licensee re-contacted the AV.

The inspector's opinion that the AV was negligent in not prescribing or discussing antibiotic therapy is an inappropriate position to take if the citation was not based on medical record review and/or discussion

In this scenario, the veterinarian instructed the staff to contact him if any problems became apparent. The staff did not follow these instructions which led to the veterinarian never being informed about the change in the animal's condition (infection). This resulted in euthanasia at the investigator's request because the animal became unusable for experiment. Adequate veterinary care was not provided because status information was not conveyed in a timely manner to the veterinarian. The appropriate citation for this circumstance would have been failure to provide a mechanism for direct and frequent communication with the attending veterinarian about the animal's condition ${ }^{3}$.

In the event a facility wishes to appeal an inspection report, the written appeal is to be submitted to the appropriate regional office within 21 days of receipt of the report ${ }^{4}$.

\section{Bernadette Juarez}

Deputy Administrator, Animal Care, APHIS, USDA. e-mail:bernadette.r.juarez@aphis.usda.gov

Published online: 24 September 2018 https://doi.org/10.1038/s41684-018-0154-0

References

1. Title 9 Chapter 1 Subpart A-Animal Welfare Section 2.33(a)(1)

2. Title 9 Chapter 1 Subpart A-Animal Welfare Section 2.33(b) (1-4)

3. Title 9 Chapter 1 Subpart A-Animal Welfare Section 2.33(b)(3)

4. https://www.aphis.usda.gov/publications/animal_welfare/2017/ AC-Tech-Note-Inspection-Report-Appeals-Process.pdf with Dr. Meyers. Further, accusations of negligence on the part of a practitioner, if valid, should be addressed with the state veterinary medical board and only used as a basis for facility citation if true negligence were established by that board.

One of the interpretations with this scenario is that the PI did not report the animal's change in condition to the facility. If he did not, then the facility would not know to contact the AV for follow-up. This is a difficult position for the facility since the PI has control of his study animals and may elect euthanasia if an animal is at risk for a decline in health or well-being and can no longer be used in the study. This is a common scenario in many facilities, including both industry and academia, wherein PIs can, and do, remove animals for a variety of reasons without having to contact veterinary resources. It is also unclear if the USDA Veterinary Medical Officer is aware that the PI has this authority, independent of veterinary opinion.

The PI's lack of communication does not mean that an inadequate program of veterinary care existed at the facility and therefore should not be the basis for a citation of non-compliance. Whether RSU will appeal the citation will be more of an administrative/political decision, but an IACUC reevaluation of the procedure for reporting adverse events is definitely a good idea. If the IACUC already has a policy it thinks is sufficiently clear, the PI is in need of training on the responsibilities of reporting adverse events and documenting activities for both facility and regulatory review.

\section{Michael Stonerook ${ }^{1 *}$ and \\ Stanley Dannemiller ${ }^{2}$}

${ }^{1}$ Lincoln Memorial University, Harrogate, Tennessee, USA. ${ }^{2}$ Northeast Ohio Medical University,

Rootstown, Ohio, USA.

*e-mail:michael.stonerook@LMUnet.edu

Published online: 24 September 2018 https://doi.org/10.1038/s41684-018-0157-x

References

1. Code of Federal Regulations, Title 9, Chapter 1, Subchapter A, Part 2-C, \$2.33, Attending Veterinarian and Adequate Veterinary Care.

2. Institute for Laboratory Animal Research. Guide for the Care and Use of Laboratory Animals. $8^{\text {th }}$ Ed. pp. 113-114 (National Academies Press, Washington, DC, 2011)

3. ACLAM Position Statement on Adequate Veterinary Care. J. Am. Assoc. Lab. Anim. Sci. 55, 826-828 (2016).

4. USDA Animal Welfare Inspection Guide. 2018. Section 6.4.2 Adequate Veterinary Care. 